\title{
Sustitución de pasta de soya por Cannavalia ensiformis en el crecimiento, sobrevivencia y actividad enzimática de tilapia (Oreochromis niloticus) (Perciforme: Cichlidae)
}

Substitution of soy paste by Cannavalia ensiformis in the growth, survival, and enzymatic activity of tilapia (Oreochromis niloticus) (Perciforme: Cichlidae)

\author{
Fanny Janet de la Cruz-Alvarado ${ }^{1}$, Carlos Alfonso Álvarez-González ${ }^{1}$, Héctor Nolasco-Soria², \\ Rafael Martínez-García ${ }^{1 *}$, Emyr Peña ${ }^{1}$, Susana Camarillo-Coop ${ }^{1}$, José Manuel Piña-Gutierrez ${ }^{1}$, \\ Bartolo Concha-Frías ${ }^{1}$
}

\begin{abstract}
RESUMEN
La harina de la leguminosa Cannavalia posee características nutricionales que la hacen apta para usarse como suplemento alimenticio en peces. Es una fuente de energía, proteína, vitaminas y minerales, pero posee factores antinutricionales que limitan su aprovechamiento. Se determinó el efecto de la inclusión de Cannavalia cocida en crecimiento, supervivencia y actividad enzimática digestiva de tilapia. Se formularon dietas para sustituir pasta de soya (PS) por harina de C. ensiformis cocida (HCe): T1 (0\% HCe-100\% PS), T2 (25\% HCe-75\% PS), T3 (50\% HCe-50\% PS), T4 (75\% HCe-25\% PS) y T5 (100\% HCe$0 \%$ PS). El mejor crecimiento en tilapia se obtuvo con T1 y resultó inversamente proporcional al porcentaje de inclusión. La supervivencia se vio afectada en el T2 (91.1\%) y T5 (94.4\%). La mayor actividad enzimática se presentó en T5 seguida de T2 en proteasas ácidas y alcalinas; la actividad de tripsina no fue significativa; la actividad de quimotripsina mostró mayor actividad enzimática en T5 y fue significante en todos los tratamientos.
\end{abstract}

PALABRAS CLAVE

Cannavalia ensiformis, crecimiento, enzimas digestivas, proteasas, Oreochromis niloticus

\begin{abstract}
Cannavalia ensiformis has nutritional characteristics as feed supplement in fish. Is a good source of energy, protein, vitamins and minerals, but it has antinutritional factors which limit its utilization. The effect of Cannavalia inclusion in the growth, survival and enzymatic activity in tilapia were determined. Soy paste was substituted (PS) for boiled C. ensiformis meal (HCe): T1 (0\% HCe-100\% PS), T2 (25\% HCe-75\% PS), T3 (50\% HCe-50\% PS), T4 (75\% HCe-25\% PS), and T5 (100\% HCe- $0 \%$ PS). Results indicated better growth of $O$. niloticus with T1. Survival was affected on T2 (91.1\%) and $100 \%(94.4 \%)$. The greatest enzymatic activity was on T5 followed by T2 on acid proteases and alkaline; thetrypsine activity was not significant; the chymotrypsinactivity showed greater enzymatic activity on T5, being significant in all treatments. Results indicated that better inclusion level of $C$. ensiformis was on $25 \%$, growth was inversely proportional to inclusion percentage.
\end{abstract}

KEYWORDS

Cannavalia ensiformis, growth, enzymes activity, proteases, Oreochromis niloticus

\footnotetext{
${ }^{1}$ Laboratorio de Acuacultura, Universidad Juárez Autónoma de Tabasco. Villahermosa, Tabasco, México.

${ }^{2}$ Laboratorio de Fisiología Comparada y Genómica Funcional, Centro de Investigaciones Biológicas del Noroeste (CIBNOR). La Paz, Baja California Sur, México.

* Autor de correspondencia. Carretera Villahermosa-Cárdenas km 0.5. 86139 Villahermosa, Tabasco, México.

E-mail: biologomartinez@hotmail.com
} 


\section{INTRODUCCIÓN}

Debido a la alta demanda de pasta de soya, existe la incertidumbre respecto de su disponibilidad a mediano plazo. Es por esto que algunos investigadores han analizado otros productos de origen vegetal como fuentes alternas de proteína en alimentos para peces (Tacon et al., 1984; Kikuchi, 1999; Cruz-Suárez et al., 2001).

Las leguminosas cuentan con contenido elevado de proteína de buena calidad, vitaminas y minerales, particularmente fósforo y hierro (Martínez-Palacios et al., 2000). Dentro de este grupo se encuentra Canavalia ensiformis (L.) DC, especie originaria de regiones tropicales (Fagbenro et al., 2007) con potencial nutricional estudiado en varias especies terrestres (Wyss y Bicjel, 1988; Udedibie, 1990; Udedibie y Nkwocha, 1990). Su aplicación en peces ha sido evaluada por varios autores (Akinbiyi, 1992; Martínez-Palacios et al., 1988, Abdo-de la Parra et al., 1998; Osuigwe et al., 2002; Fagbenro et al., 2007; Okomoda et al., 2016; Tiamiyu et al., 2016). Sin embargo, su enorme potencial nutritivo se ve reducido por todo un conjunto de factores antinutricionales (FAN), que obstruyen el aprovechamiento de sus nutrientes y limitan su uso en la elaboración de dietas para peces (Martínez-Palacios et al., 1988; Abdo de la Parra et al., 1998; Osuigwe et al., 2002; Fagbenro et al., 2007; Akande y Fabiyi, 2010). Los FAN que se encuentran en esta especie son: la canavanina (Rosenthal, 1992), concanavalina A y B (lectinas), inhibidores de proteasas, fitatos y ácidos oxálicos que afectan significativamente el crecimiento y procesos fisiológicos, e incluso llegan a provocar la mortalidad en los peces (Alegbeleye et al., 2001).

Considerando lo anterior, Okomoda et al. (2016) y Tiamiyu et al. (2016) han obtenido buenos resultados de eliminación de FAN utilizando procesos térmicos en semillas de C. ensiformis, lo cual incrementa la biodisponibilidad de los nutrientes. Sin embargo, es necesario determinar el efecto de $C$. ensiformis en Oreochromis niloticus para estipular su factibilidad de inclusión dietaria. También es importante apoyarse en análisis sobre la actividad enzimática digestiva del pez, que ayuden a mejorar la nutrición animal (Köprücü y Ozdemir, 2005), ya que un alimento que no puede ser digerido es de poco valor nutricional (Köprücü y Ozdemir, 2005). A este respecto, la actividad de la pepsina, tripsina y quimotripsina se utiliza como un indicador de la capacidad digestiva del pez (Moyano et al., 1996).

La presente investigación se diseñó para determinar el efecto en parámetros productivos de los diferentes niveles de sustitución de la pasta de soya por harina C. ensiformis cocida, así como su efecto en la actividad de proteasas digestivas de O. niloticus, con la finalidad de evaluar su inclusión como fuente de proteína alterna para el cultivo de la tilapia.

\section{Materiales y MÉtodos}

Este estudio se llevó a cabo en el Laboratorio de Acuicultura Tropical de la División Académica de Ciencias Biológicas, perteneciente a la Universidad Juárez Autónoma de Tabasco (UJAT). Se evaluó el crecimiento, la supervivencia y la actividad de enzimas digestivas en juveniles de $O$. niloticus usando cinco diferentes niveles de sustitución de pasta de soya por harina cocida de C. ensiformis.

\section{Obtención de organismos}

Para este experimento, los juveniles de tilapia se obtuvieron de un lote de reproductores de 150 hembras (150 g en promedio) y 50 machos (200 g en promedio) mantenidos en un tanque rectangular de concreto de $300 \mathrm{~m}^{3}$, localizado en el área de reproducción de tilapia del Laboratorio de Acuicultura Tropical. Las crías se obtuvieron de la colecta de embriones para la cual se utilizó un cedazo de plástico de $1 \mathrm{~mm}$ de luz malla y fueron colocadas en un estanque circular de plástico de 2000 L, donde, durante 28 días, se les proporcionó un alimento comercial hormonado para tilapia con $52 \%$ de proteína y 14\% de lípidos (El Pedregal Silver $\mathrm{Cup}^{\circledR}$, Toluca, México) que contenía una dosis de 60 $\mathrm{mg} / \mathrm{kg}$ de $17 \alpha$-metiltestosterona (MT) para promover su reversión sexual. Finalmente, los peces fueron sembrados en un sistema de recirculación para iniciar el experimento.

\section{Diseño experimental}

El experimento consistió en la sustitución parcial de pasta de soya (PS) por harina de C. ensiformis cocida (HCe), en cinco niveles de inclusión: T1 (0\% HCe-100\% PS), T2 (25\% HCe-75\% PS), T3 (50\% HCe-50\% PS), T4 (75\% HCe-25\% PS) y T5 (100\% HCe-0\% PS), respectivamente. Éstos fueron evaluados por triplicado en un sistema de recirculación que constó de 15 unidades experimentales de $100 \mathrm{~L}$ de capacidad conectados a una bomba centrífuga de $1 / 3$ HP (Jacuzzi Star-rite $^{\circledR}$, JWPA5D-230 ${ }^{\text {a }}$ Delavan, Winsconsin, USA), un filtro de arena (STA-RITE ${ }^{\circledR}$ S166T, Delavan, Winsconsin, USA), una lámpara de UV de 25 Watts 
(Emperor Aquatics ${ }^{\circledR}, 02025$, Pottstown, USA), un termostato de titanio (PSA ${ }^{\circledR}$, R9CE37I, Delavan, USA) y un reservorio de concreto de $2000 \mathrm{~L}$ de capacidad. Se colocaron 15 alevines por unidad experimental con peso promedio de $0.972 \pm 0.05 \mathrm{~g}$ y una longitud promedio de $3.84 \pm 0.15 \mathrm{~cm}$, distribuidos aleatoriamente, con un total de 225 alevines de tilapia del Nilo O. niloticus. Durante el experimento, los peces fueron alimentados cuatro veces al día (9:00, 11:00, 13:00 y 15:00 h) con una ración equivalente a 10\% de su biomasa. La concentración de oxígeno $(6.24 \pm 0.24$ $\mathrm{mg} / \mathrm{l})$ y la temperatura $\left(28.72 \pm 0.16^{\circ} \mathrm{C}\right)$ se midieron con uno oxímetro $\mathrm{YSI}^{\circledR} 55$ (con precisión de $0.1{ }^{\circ} \mathrm{C}$ y $0.01 \mathrm{mg} / \mathrm{l}$, California, USA) y el $\mathrm{pH}(7.2 \pm 0.23)$ con un potenciómetro (Hanna Instruments ${ }^{\circledR}$, HI 98311, Rhode Island, USA). La red de aireación contenía una piedra porosa por unidad experimental, alimentada por un aireador (Sweetwater, 0.5 HP). La limpieza de las unidades se realizó mediante sifón tres veces al día para retirar las excretas. Las biometrías se llevaron a cabo cada 15 días utilizando aceite de clavo a una concentración de $0.1 \mathrm{~mL} / \mathrm{L}$ de agua como anestésico. Los peces se dejaron en inanición $24 \mathrm{~h}$ antes de la biometría.

\section{Procesamiento de la harina y formulación de las dietas}

Para el procesamiento de cocción de la harina de C. ensiformis, se pesaron $200 \mathrm{~g}$ de semillas de C. ensiformis; luego, se colocaron en un matraz Erlenmeyer y se hidrataron con $1 \mathrm{~L}$ de agua destilada durante $12 \mathrm{~h}$ a temperatura ambiente. Después, se procedió a decantar y lavar con agua destilada. Posteriormente, se llevaron a ebullición durante 30 minutos usando una relación frijol: agua de 1:5 (Anduaga-Cota et al., 2002). El frijol se decantó y se secó en una estufa (VWR ${ }^{\circledR}$, modelo 1680) a temperatura de $70{ }^{\circ} \mathrm{C}$ por $24 \mathrm{~h}$; seguidamente, se molieron las semillas en un pulverizador (PULVEX ${ }^{\circledR}$ 200, México, D. F.) tamizando a 500 micras y se almacenaron a 4 ${ }^{\circ} \mathrm{C}$. Posteriormente, se formularon las cinco dietas isocalóricas e isolipídicas utilizando la harina de $C$. ensiformis cocida por medio del programa MIXITWIN V. 5.0 como se muestra en el cuadro 1.

\section{Parámetros de crecimiento y supervivencia}

El experimento tuvo una duración de 45 días y, al final de éste, se determinaron la ganancia absoluta en peso (AWG, g pez $\left.z^{-1}\right)=W f(g)-W i(g)$; la tasa de crecimiento específico $(\mathrm{SGR}, \%)=100(\ln W \mathrm{f}-\ln W \mathrm{i}) / \mathrm{T}$, donde: Wi y Wf son el peso inicial y final del pez, respectivamente, y $\mathrm{T}$ es el número de días en el periodo de alimentación; el factor de conversión de alimento $(\mathrm{FCR})=$ alimento seco entregado $(\mathrm{g}) /$ ganancia en peso húmedo (g); la eficiencia del alimento $\mathrm{FE}=$ (peso final peso inicial)/ alimento consumido y la supervivencia $(\%)=$ organismos finales/organismos iniciales $\times 100$.

\section{Preparación de extractos multienzimáticos}

Al terminar el bioensayo, los peces se mantuvieron en inanición por $24 \mathrm{~h}$ para eliminar el exceso de heces y el alimento del tracto digestivo. Al día siguiente se sacrificaron por shock térmico ocho juveniles por unidad experimental y se les extrajo el estómago y el intestino sobre una placa fría a una temperatura de $4{ }^{\circ} \mathrm{C}$ para evitar desnaturalización enzimática. La metodología para esta preparación se apega a la propuesta por Saunders et al. (1972), que más tarde fue modificada por Dimes y Haard (1994). Las muestras de los intestinos fueron homogenizadas a partir de cada réplica experimental en el buffer Tris- $\mathrm{HCl} 50 \mathrm{mM}$ $+\mathrm{CaCl}_{2} 25 \mathrm{mM}, \mathrm{pH}$ 7.5. Por su parte, los estómagos se homogenizaron con buffer Glicina- $\mathrm{HCl}$ al $0.1 \mathrm{M}$, $\mathrm{pH}$ 2.0. Las muestras se centrifugaron a $12,000 \mathrm{rpm}$ por $30 \mathrm{~min}$ a $4{ }^{\circ} \mathrm{C}$; se les extrajo el sobrenadante y se almacenaron a $-20^{\circ} \mathrm{C}$ hasta el momento de su análisis.

\section{Actividad enzimática}

Para medir la actividad específica de las proteasas digestivas, se utilizaron las técnicas que se describen a continuación.

En proteasas alcalinas, se utilizó el método descrito por Walter (1984) usando caseína (1\%) como sustrato con una solución amortiguadora Tris- $\mathrm{HCl} 50 \mathrm{mM}$, $\mathrm{CaCl}_{2} 20 \mathrm{mM}, \mathrm{pH}$ 7.5. Para proteasas ácidas, la técnica descrita por Anson (1938) usando hemoglobina (1\%) como sustrato y sustituyendo las soluciones amortiguadoras de Tris- $\mathrm{HCl} 50 \mathrm{mM}, \mathrm{CaCl}_{2} 20 \mathrm{mM}, \mathrm{pH} 7.5$ por Glicina- $\mathrm{HCl} 0.1 \mathrm{M}$ a pH 2.0. A una temperatura de $37^{\circ} \mathrm{C}$, el tiempo de incubación en ambos casos fue de 15 minutos para las proteasas ácidas y de 10 minutos para las alcalinas. Todos los ensayos se hicieron por triplicado.

Para los análisis de actividad de tripsina, se empleó el método de Erlanger et al. (1961) usando como sustrato BAPNA $1 \mathrm{mM}(\mathrm{N}-\alpha$ - benzoil-DL-arginina 4-nitroanilida) en buffer Tris- $\mathrm{HCl} 50 \mathrm{mM}+\mathrm{CaCl}_{2} 10$ $\mathrm{mM}$ a $\mathrm{pH}$ 8.2. El BAPNA fue previamente diluido en $100 \mu \mathrm{l}$ de dimetilsulfoxido (DMSO). Para iniciar la reacción, se mezclaron $560 \mu \mathrm{l}$ del sustrato con $10 \mu \mathrm{l}$ 
Cuadro 1. Formulación de las dietas experimentales, sustituyendo la pasta de soya con harina de Cannavalia ensiformis cocida usadas durante la evaluación in vivo en Oreochromis niloticus.

\begin{tabular}{|c|c|c|c|c|c|}
\hline $\begin{array}{l}\text { INGREDIENTE } \\
\text { (G } 100 \text { G }^{-1} \text { DIETA) }\end{array}$ & ${ }_{100} \%$ PS-0 $\%$ HCE & ${ }_{75} \%$ PS- $25 \%$ HCE & $\begin{array}{c}50 \% \text { PS- } \\
50 \% \text { HCE }\end{array}$ & $\begin{array}{c}25 \% \text { PS- } \\
75 \% \text { HCE }\end{array}$ & $0 \% \mathrm{PS}-100 \% \mathrm{HCE}$ \\
\hline Harina de soya $44 \%$ a & 315 & 231 & 156 & 81 & 0 \\
\hline Harina C. ensiformis cocida ${ }^{c}$ & 0 & 99 & 195 & 292.5 & 390 \\
\hline Harina de sorgo $9 \%$ & 375 & 352.5 & 331.5 & 309 & 285 \\
\hline Harina de carne $50 \%{ }^{a}$ & 390 & 390 & 390 & 390 & 390 \\
\hline H. de pescado ${ }^{a}$ & 210.0 & 217.5 & 217.5 & 217.5 & 225.0 \\
\hline Aceite de pescado ${ }^{d}$ & 90 & 90 & 90 & 90 & 90 \\
\hline Aceite de soya ${ }^{e}$ & 45 & 45 & 45 & 45 & 45 \\
\hline Grenetina $^{\mathrm{f}}$ & 30 & 30 & 30 & 30 & 30 \\
\hline Premezcla de vitaminas ${ }^{\mathrm{g}}$ & 22.5 & 22.5 & 22.5 & 22.5 & 22.5 \\
\hline Premezcla de minerales ${ }^{g}$ & 15 & 15 & 15 & 15 & 15 \\
\hline Vitamina $C^{\text {h }}$ & 7.5 & 7.5 & 7.5 & 7.5 & 7.5 \\
\hline \multicolumn{6}{|c|}{ Análisis químico (g / 100 g materia seca) } \\
\hline Proteína (\%) & 52.64 & 50.42 & 48.89 & 47.73 & 46.50 \\
\hline Extracto etéreo (\%) & 19.11 & 18.34 & 17.55 & 16.76 & 14.23 \\
\hline Fibra cruda (\%) & 0.37 & 0.73 & 1.83 & 2.69 & 2.79 \\
\hline Cenizas (\%) & 12.22 & 11.92 & 11.37 & 10.93 & 10.50 \\
\hline Extracto libre de $\mathrm{N}(\%)$ & 15.67 & 18.58 & 20.36 & 21.89 & 25.99 \\
\hline Energía (cal/g) & 5146.69 & 5071.57 & 5020.13 & 4998.22 & 4953.92 \\
\hline
\end{tabular}

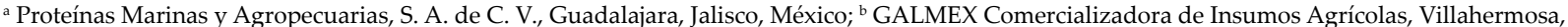

Tabasco, México; ${ }^{\mathrm{c}}$ Empresa comercializadora Teapa, Tabasco, México; ${ }^{\mathrm{d}}$ Sigma - Aldrich \# catálogo F-8020; ${ }^{\mathrm{e}}$ Pronat Ultra, Mérida, Yucatán, México; ${ }^{\mathrm{f}} \mathrm{D}^{\prime}$ gari, Productos alimenticios y dietéticos relámpago, S. A. de C. V., Tlalpan, México D. F.; g Pedregal (para trucha Silver Cup), Toluca, Estado de México; ${ }^{\mathrm{h}}$ México; ROVIMIX R C-EC (Roche) agente activo de 35\%.

del extracto enzimático y se incubó el complejo por 5 minutos a $37{ }^{\circ} \mathrm{C}$. La reacción se detuvo adicionando $160 \mu \mathrm{l}$ de ácido acético a 30\%. La absorbancia fue medida a $410 \mathrm{~nm}$. El ensayo se realizó por triplicado.

En el caso de quimotripsina, se determinó por el método de Asgeirsson y Bjarnason (1991) usando como sustrato BTEE (N-benzoil-L-tirosina etilester) $5 \mathrm{mM}$ en buffer Tris- $\mathrm{HCl} 44.4 \mathrm{mM}+\mathrm{NaCl} 55.5 \mathrm{mM} \mathrm{pH}$ 7.8. Se colocaron $623 \mu \mathrm{l}$ de buffer directamente en la celda de cuarzo del espectrofotómetro y se calibró a cero. Posteriormente, se agregaron $70 \mu \mathrm{l}$ del sustrato y se leyó la absorbancia a $256 \mathrm{~nm}$ cada 20 segundos durante 2 minutos. Transcurrido este tiempo, se agregaron $10 \mu \mathrm{l}$ de extracto multienzimático y nuevamente se leyó la absorbancia cada $20 \mathrm{~s}$ por $2 \mathrm{~min}$. El ensayo se realizó por quintuplicado; el delta de absorbancia se calculó como la diferencia entre la absorbancia de la reacción catalizada y la absorbancia del sustrato una vez que ambos se habían estabilizado.

\section{Análisis estadístico}

Para la evaluación del crecimiento en peso se utilizó un análisis de varianza de una vía, al cumplir los postulados de normalidad y homoscedasticidad. Para determinar las diferencias significativas entre los tratamientos, se utilizó la prueba a posteriori de Tukey. Los índices de eficiencia alimentaria, las variables de crecimiento y supervivencia y las actividades enzimáticas fueron evaluados con una prueba de Kruskal-Wallis. Posteriormente, cuando se encontró diferencia significativa, se realizó una prueba a posteriori de Nemenyi, usando un valor de significancia de 0.05 . El programa estadístico ocupado fue el STATISTICA ${ }^{\mathrm{TM}} \mathrm{v}$ 7.0.

\section{RESULTADOS Y DISCUSIÓN}

Terminado el experimento de crecimiento (45 días), se observó una supervivencia de 100\% en T1, T2 y T3, 
Cuadro 2. . Índices de eficiencia alimentaria (IEA), variables de crecimiento y porcentaje de supervivencia de alevines de O. niloticus alimentados con las dietas de harina de Cannavalia ensiformis cocida (promedio $\pm \mathrm{DE}$ ).

\begin{tabular}{|c|c|c|c|c|c|}
\hline \multicolumn{6}{|c|}{ Porcentaje de inclusión de harina cocida de C. ensiformis (\%) } \\
\hline ÍNDICES & ${ }_{100} \% \mathrm{PS}-0 \% \mathrm{HCE}$ & $75 \%$ PS- $25 \%$ HCE & $\begin{array}{c}50 \% \text { PS- } \\
50 \% \text { HCE }\end{array}$ & $\begin{array}{c}25 \% \text { PS- } \\
75 \% \text { HCE }\end{array}$ & $0 \%$ PS- $100 \%$ HCE \\
\hline Wi & $0.94 \pm 0.10$ & $0.97 \pm 0.15$ & $0.94 \pm 0.02$ & $1.06 \pm 0.06$ & $0.95 \pm 0.10$ \\
\hline Wf & $9.16 \pm 0.83$ & $5.27 \pm 0.97$ & $4.19 \pm 0.25$ & $4.29 \pm 0.23$ & $3.86 \pm 0.48$ \\
\hline LF & $8.13 \pm 0.34$ & $6.85 \pm 0.44$ & $6.24 \pm 0.05$ & $6.26 \pm 0.08$ & $6.13 \pm 0.22$ \\
\hline AWG & $8.22 \pm 0.81$ & $4.31 \pm 0.82$ & $3.25 \pm 0.23$ & $3.23 \pm 0.20$ & $2.92 \pm 0.38$ \\
\hline FCR & $4.42 \pm 0.14$ & $2.07 \pm 0.04$ & $2.38 \pm 0.21$ & $2.68 \pm 0.20$ & $2.54 \pm 0.07$ \\
\hline SGR & $5.43 \pm 0.27$ & $4.03 \pm 0.07$ & $3.56 \pm 0.10$ & $3.33 \pm 0.12$ & $3.35 \pm 0.04$ \\
\hline SR (\%) & 100 & 100 & 100 & 91.1 & 94.4 \\
\hline K & $1.705 \pm 0.09$ & $1.633 \pm 0.03$ & $1.726 \pm 0.09$ & $1.748 \pm 0.07$ & $1.675 \pm 0.03$ \\
\hline FE & $0.23 \pm 0.01$ & $0.48 \pm 0.01$ & $0.42 \pm 0.04$ & $0.38 \pm 0.03$ & $0.39 \pm 0.01$ \\
\hline
\end{tabular}

Ganancia absoluta en peso (AWG, g pez-1) = Wf (g) - Wi (g); la tasa de crecimiento específico (SGR, \%) = 100 (lnWf - $\operatorname{lnWi}$ ) / T, donde: Wi y Wf son el peso inicial y final del pez, respectivamente, y T es el número de días en el periodo de alimentación; el factor de conversión de alimento (FCR) = alimento seco entregado $(\mathrm{g}) /$ ganancia en peso húmedo $(\mathrm{g})$; el factor de condición $(\mathrm{K})=(($ peso final $(\mathrm{g}) /$ longitud estandar $(\mathrm{cm}) 3 \times 100$; la eficiencia del alimento $\mathrm{FE}=(\mathrm{peso}$ final - peso inicial) / alimento consumido y supervivencia SR $(\%)=$ organismos finales / organismos iniciales $\times 100$.

mientras que T4 y $\mathrm{T} 5$ presentaron un 91.1 y $94.4 \%$ de supervivencia, respectivamente. Los resultados de los parámetros Wf, Lf, AWG y SGR de los tratamientos T1 y T2 fueron estadísticamente significativos $(p<0.05)$ con respecto al resto de los tratamientos. El T2 obtuvo valores de FCR $(2.07 \pm 0.04)$ y FE $(0.48 \pm$ $0.01)$, los cuales fueron estadísticamente significativos con respecto a los demás tratamientos de inclusión de $C$. ensiformis cocida $(p<0.05)$ (cuadro 2). En todos los tratamientos, los valores alcanzaron su mayor rendimiento conforme decrecían los niveles de concentración de $C$. ensiformis cocida en la dieta, de manera que existió una relación indirecta entre la cantidad de C. ensiformis cocida contenida en la dieta y el crecimiento de O. niloticus (figura 1).

En cuanto a la actividad enzimática, la mayor actividad de las proteasas ácidas se presentó en el T1, seguido de T2 y T3, con diferencias estadísticamente significativas $(p<0.05)$ entre estos tratamientos (figura 2). Las proteasas alcalinas mostraron mayor actividad en T1 seguidas de T2, con diferencias significativas entre ellas $(p<0.05)$ (figura 3). Con respecto a la tripsina, no hubo diferencias significativas entre los tratamientos (figura 4), mientras que, en quimotripsina, se presentó una mayor actividad en T5 seguida de T4, T3 y T2, con diferencias estadísticamente significativas $(p<0.05)$ (figura 5).

La cannavalia es una leguminosa con un alto potencial como fuente de energía, proteínas, vitaminas

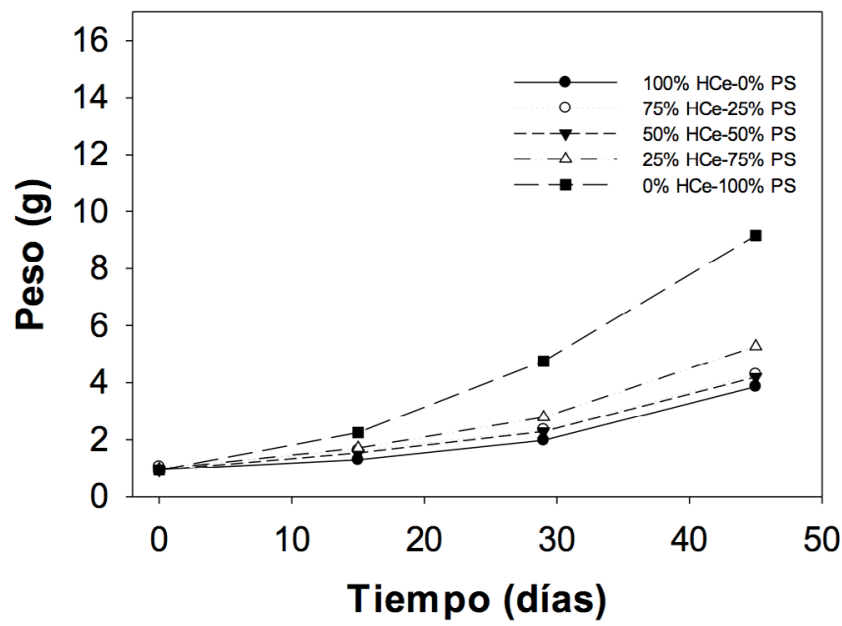

Figura 1. Crecimiento en peso promedio $(g \pm D E)$ de alevines de Oreochomis niloticus alimentados con las dietas de harina de Cannavalia ensiformis cocida.

y minerales para el cultivo de peces. Sin embargo, presenta algunos FAN que limitan su utilización en la elaboración de dietas para peces (Fagbenro et al., 2007; Akande y Fabiyi, 2010; Anyanwu et al., 2012). El efecto de los antinutrientes presentes en las fuentes de proteínas vegetales ya ha sido reportado por Alarcón et al. (1999), Moyano-López et al. (1999), El-Sayed et al. (2000), Francis et al. (2001), Chong et al. (2002), Pérez et al. (2003), Elemo et al. (2011), cuyos resultados son muy variados y dependen del tipo de leguminosa, 


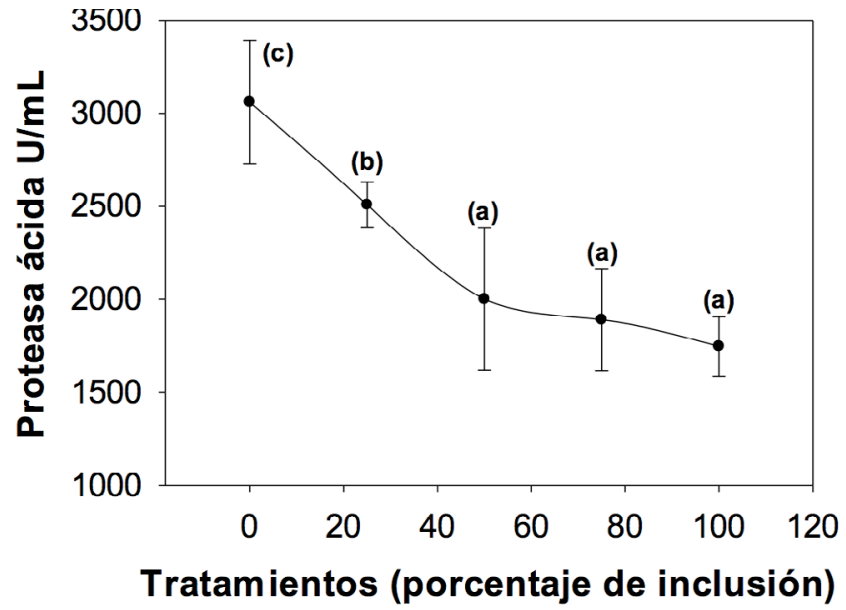

Figura 2. Actividad enzimática digestiva de proteasas ácidas en alevines de Oreochomis niloticus alimentados con las dietas de harina de Cannavalia ensiformis cocida.

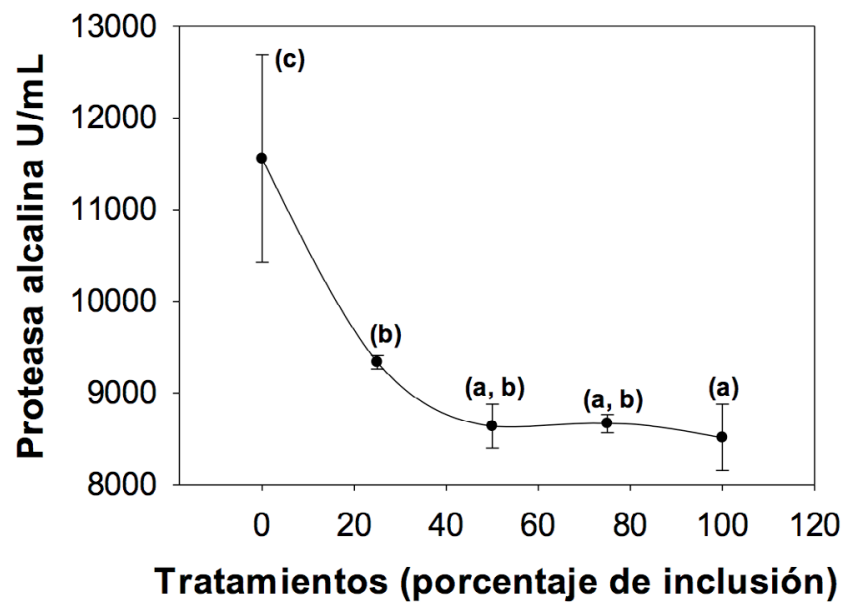

Figura 3. Actividad enzimática digestiva de proteasas alcalina en alevines de Oreochomis niloticus alimentados con las dietas de harina de Cannavalia ensiformis cocida.

ya sea por la presencia de sustancias que limitan la acción de las enzimas sobre su sustrato (lectinas) o la presencia de inhibidores como los antitripsinógenos, entre otros.

En este estudio se observó que el crecimiento de O. niloticus disminuía con respecto al incremento de inclusión de harina C. ensiformis en la dieta, lo cual ya ha sido reportado por Martínez-Palacios et al. (1988), Akinbiyi (1992), Abdo De la Parra et al. (1998), Olvera-Novoa y Olivera-Castillo (2000), Osuigwe et al. (2002), Anyanwu et al. (2012), Okomoda et al. (2016), Tiamiyu et al. (2016). Sin embargo y debido a la calidad nutrimental de las harinas de leguminosas, se han utilizado diferentes métodos para la eliminación

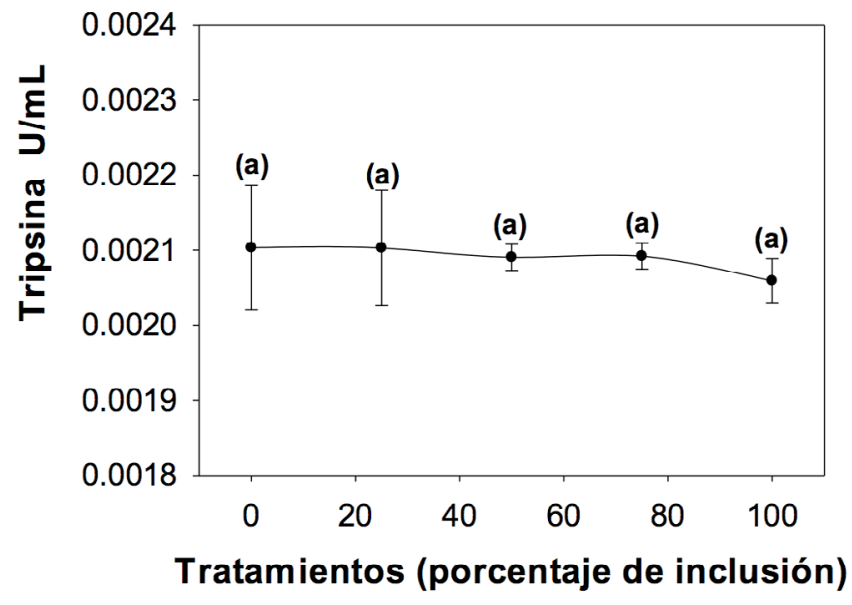

Figura 4. Actividad enzimática digestiva de tripsina en alevines de Oreochomis niloticus alimentados con las dietas de harina de Cannavalia ensiformis cocida.

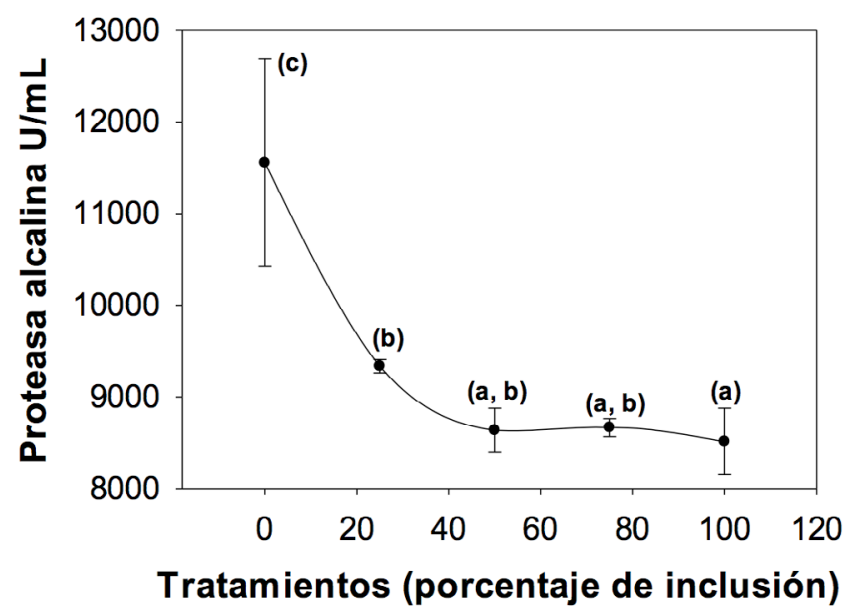

Figura 5. Actividad enzimática digestiva de quimotripsina en alevines de Oreochomis niloticus alimentados con las dietas de harina de Cannavalia ensiformis cocida.

de antinutrientes; entre ellos, el tratamiento térmico (Garg et al., 2002; Thiessen et al., 2003; Urbano et al., 2003; Undesi et al., 2007; Akande y Fabiyi, 2010; Ndidi et al., 2014; Okomoda et al., 2016; Tiamiyu et al., 2016) y el tratamiento con solventes de ácido orgánico en la harina (Das-Purkayastha et al., 2014). A partir de éstos, se ha recomendado una sustitución de $25 \%$ de C. ensiformis para dietas en peces; sin embargo, no existen estudios enfocados en el efecto de esta harina sobre la actividad enzimática de los peces en estudio.

Las actividades enzimáticas de proteasas ácidas y alcalinas mostraron una disminución respecto al aumento del porcentaje de inclusión de $C$. ensiformis. Esto es similar a los reportes realizados por Francis et 
al. (2001), quienes reportan que la diminución de la actividad de proteasas alcalinas podría deberse a que la retroalimentación positiva pancreática esté siendo afectada por los inhibidores de la fuente vegetal como un efecto post-ingesta. La tripsina presentó una actividad constante en todos los tratamientos, lo cual puede indicar que el tratamiento térmico al que se sometió la leguminosa C. ensiformis fue suficiente para disminuir algunos componentes antinutricionales de inhibición de tripsina (Akande et al., 2010; Doss et al., 2011; Okomoda et al., 2016; Tiamiyu et al., 2016). No obstante, también puede deberse a una sobreregulación de tripsina como una necesidad del pez de aprovechar los nutrientes presentes en la dieta, pues la proteína dietaria puede estimular al páncreas para regular la secreción de tripsina (Bakke et al., 2011; Rodiles et al., 2012; García-Meilán et al., 2013), la cual participa en la degradación proteica y en la liberación de aminoácidos utilizados en el crecimiento, reproducción y energía de los peces (Al-Saraji y Nasir, 2013). Por otro lado, la actividad de quimotripsina incrementó conforme aumentaba el porcentaje de inclusión de $C$. ensiformis, resultados que también pueden deberse a que muchos peces tienden la capacidad de contrarrestar la presencia de inhibidores de proteasas incrementando la producción de tripsina y quimotripsina (Francis et al., 2001).

\section{Conclusiones}

El mejor nivel de inclusión de Cannavalia ensiformis cocida corresponde a $25 \%$. Los FAN tienen efecto adverso sobre la actividad enzimática de $O$. niloticus cuando se sobrepasan esos valores, lo que se refleja en el crecimiento que resulta inversamente proporcional al porcentaje de inclusión de $C$. ensiformis. Se recomiendan más análisis de tratamientos químicos y físicos que permitan eliminar los FAN no termoestables y con ello sustituir la harina de pasta de soya en dietas experimentales, como fuentes proteína alterna hacia el diseño de dietas para $O$. niloticus.

\section{Agradecimientos}

AlConsejo Nacional de Ciencia y Tecnología (Conacyt) y a la Organización de Estados Americanos (OEA), por el apoyo mediante la beca otorgada durante la realización de esta investigación. Al Centro de Investigaciones Biológicas del Noroeste (CIBNOR), donde se realizó estancia de investigación. Al proyecto "Fortalecimiento de la Maestría en Ciencias Ambientales para su Permanencia en el Padrón Nacional de Posgrados de Calidad del Conacyt", clave: TAB-2014C29-245836", por el apoyo para la realización de la estancia de investigación. 


\section{LITERATURA CITADA}

Abdo de la Parra, M. I., C. A. Martínez-Palacios, I. E. Martínez-Rodríguez, B. González-Rodríguez, M. A. Olvera, M. L. Vásquez, C. Chávez-Sánchez.1998. Advances in the use of the bean Canavalia maritima as a possible source of protein in diets for tilapia (Oreochromis niloticus). In: H. Grizel, P. Kestemont (eds.). Abstracts of the International Conference - Aquaculture Europe ‘98. European Aquaculture Society, Special Publication No. 26. Oostende, Belgium. pp. 2-3.

Akande, K. E., E. F. Fabiyi. 2010. Effect of processing methods on some antinutritional factors in legume seeds for poultry feeding. International Journal of Poultry Science 9(10): 996-1001.

Akinbiyi, A. 1992. The use of thermally-processed jack bean (Canavalia ensiformis) in Nile tilapia diets. M. Sc. Thesis Dissertesion, University of Ibadan, Nigeria. 70 pp.

Alarcón, F. J., F. J. Moyano, M. Díaz. 1999. Effect inhibitors presents in protein sources on digestive proteases of juvenile sea bream (Sparus aurata). Aquatic Living Resources 12(4): 233-238.

Alegbeleye, W. O., A. Oresegun, O. D. Ajimoti. 2001. An assessment of Jack bean (Canavalia ensiformis) meal as an ingredient in the diets for Clarias gariepinus (Burche11 1822) fingerlings. In: Eyo A. A. (ed.). Fish Nutrition and Fish Feed Technology. pp. 92-97. Proceedings of a National Symposium held at the National Institute for Oceanography and Marine Research (NIOMR), Lagos, Nigeria, October 1999, Proceedings Series No. 1 NIOMR, Lagos.

Al-Saraji, A. Y. J., N. A. N. Nasir. 2013. Effect of different dietary proteins and fats on the digestive enzymes activities in the common carp fingerlings (Cyprinus carpio L.) reared in floating cages. Mesopotamian Journal of Marine Science 28(2): 121-130.

Anduaga-Cota, R., A. G. Cota-Gastélum, M. R. Falcón-Villa, G. Yánez-Farías, M. J. Barrón-Hoyos. 2002. Medición de dureza en frijol cocido con una celda de extrusión por alambres: propuesta de una celda de menor tamaño. Memorias del IV Congreso del Noroeste en Ciencias Alimentarias y Biotecnología. Hermosillo, México.

Anson, M. L. 1938. The estimation of pepsin, trypsin, papain and cathepsin with hemoglobin. The Journal of General Physiology 22: 79-89.

Anyanwu, D. C., E. N. Umeh, J. I. Offor. 2012. Growth and nutrient utilization of Clarias gariepinus fed dietary levels of jackbean (Canavalia ensiformis) meal. Pakistan Journal of Nutrition 11(11): 1033-1036.

Asgeirsson, B., J. B. Bjarnason. 1991. Structural and kinetic properties of chymotrypsin from Atlantic cod (Gadusmorhua). Comparison with bovine chymotrypsin. Comparative Biochemistry and Physiology 99B (2): 327-335.

Chong, A. S. C., G. R. Hashim, A. B. Ali. 2002. Assessment of dry matter and protein digestibilities of selected raw ingredients by discus fish (Symphysodon aequifasciata) using in vivo and in vitro methods. Aquaculture Nutrition 8: 229-238.
Cruz-Suárez, L. E., D. Ricque-Marie, M. Tapia-Salazar, I. M. Mc Callum, D. Hickling. 2001. Assessment of differently processed feed pea (Pisumsativum) meals and canola meal (Braica sp.) in diets for bleu shrimp (Litopenaeussty lirostris). Aquaculture 196: 87-104.

Das-Purkayastha, M., J. Gogoi, D. Kalita, P. Chattopadhyay, K. S. Nakhuru, D. Goyary, C. L. Mahanta. 2014. Physicochemical and functional properties of rapeseed protein isolate: influence of antinutrient removal with acidified organic solvents from rapeseed meal. Journal of Agricultural and Food Chemistry 62(31): 7903-7914.

Doss, A., M. Pugalenthi, V. G. Vadivel, G. Subhashini, R. A. Subash. 2011. Effects of processing technique on the nutritional composition and antinutrients content of under utilized food legume Canavalia ensiformis L.DC. International Food Research Journal 18(3): 965-970.

Dimes, L. E., N. Haard. 1994. Estimation of protein digestibility: Development of an in vitro method for estimating protein digestibility in salmonids. Comparative Biochemistry and Physiology Part A: Physiology 108: 349-362.

Elemo, G. N., B. O. Elemo, O. L. Erukainure, 2011. Activities of some enzymes, enzyme inhibitors and antinutritional factors from the seeds of sponge gourd (Luffa aegyptiaca M.). African Journal of Biochemistry Research 5(3): 86-89.

El-Sayed, A. F. M., I. M. Martínez, F. J. Moyano. 2000. Assessment of the effect of plant inhibitors on digestive proteases of Nile Tilapia using in vitro assays. Aquaculture International 8: 403-415.

Erlanger, B., N. Kokowsky, W. Cohen. 1961. The preparation and properties of two new chromogenic substrates of trypsin. Archives of Biochemistry and Biophysics 95: 271-278.

Fagbenro, O. A., E. O. Adeparusi, W. A. Jimoh. 2007. Evaluation and nutrient quality of detoxified jackbean ( $\mathrm{Ca}$ navalia ensiformis) seeds, cooked in distilled water or trona solution, as a substitute for soybean meal in Nile tilapia, Oreochromis niloticus, diets. Journal of Applied Acuaculture 19(2): 83-100.

Francis, G., H. Makkar, K. Becker. 2001. Antinutrional factors present in plant-derived alternate fish feed ingredients and their effects in fish. Aquaculture 199: 197-227.

García-Meilán, I., J. M. Valentín, R. Fontanillas, M. A. Gallardo. 2013. Different protein to energy ratio diets for gilthead sea bream (Sparus aurata): Effects on digestive and absorptive processes. Aquaculture 412(413): 1-7.

Garg, S. K., A. Kalla, A. Bhatnagar. 2002. Evaluation of raw and hydrothermically procesed leguminous seeds as supplementary feed for the growth of two Indian major carp species. Aquaculture Research 33: 151-163.

Kikuchi, K. 1999. Use of defatted soybean meal as a substitute for fish meal in diets of Japanese flounder (Paralichthys olivaceus). Aquaculture 179: 3-11. 
Köprücü, K., Y. Özdemir. 2005. Apparent digestibility of selected feed ingredients for Nile tilapia (Oreochromis niloticus). Aquaculture 250: 308-316.

Martínez-Palacios, C. A., R. Galván-Cruz, M. A. Olvera-Novoa, C. Chávez-Martinez. 1988. The use of jack bean (Cannavalia ensiformis Leguminosae) meal as a partial substitute for fish in diets for tilapia (Oreochromis mossambicus Cichlidae). Aquaculture 68: 165-175.

Martínez-Palacios, C. A., M. C. Chávez-Sánchez, M. A. Olvera-Novoa, M. I. Abdo de la Parra. 2000. Fuentes alternativas de proteínas vegetales como substitutos de la harina de pescado para la alimentaciónen acuicultura. Unidad de Investigación y Desarrollo Tecnológico en Acuicultura y Manejo Ambiental del CIAD, A.C. Mazatlán, Sinaloa, México. Avances en Nutrición Acuícola III. pp. 279-324.

Moyano, F. J., M. Díaz, F. J. Alarcon, M. C. Sarasquete. 1996. Characterization of digestive enzyme activity during larval development of gilthead seabream (Sparus aurata). Fish Physiology Biochemistry 15: 121-130.

Moyano-López, F. J., M. Díaz, F. J. Alarcón-López. 1999. Inhibition of digestive proteases by vegetable meals in three fish species; seabream (Sparus aurata), tilapia (Oreochromis niloticus) and African sole (Soleas enegalensis). Comparative Biochemistry and Physiology Part B 122: 327-332.

Ndidi, U. S., C. U. Ndidi, A. O. Aliyu, M. F. G. Billy, O. Okpe. 2014. Proximate antinutrients and mineral composition of raw and processed (boiled and roasted) Sphenostylis stenocarpa seeds from southern Kaduna, Northwest Nigeria. ISRN Nutrition Article ID 280837: 1-9.

Okomoda, V. T., L. O. Tiamiyu, S. G. Uma. 2016. Effects of hydrothermal processing on nutritional value of $\mathrm{Ca}$ navalia ensiformis and its utilization by Clarias gariepinus (Burchell, 1822) fingerlings. Journal of Aquaculture Engineering and Fisheries Research 3(1): 214-219.

Olvera-Novoa, M. A., L. Olivera-Castillo. 2000. Potencialidad del uso de las leguminosas como fuente proteica en alimentos para peces. Pp. 327-348. En: Civera-Cerecedo, R., C. J. Pérez-Estrada, D. Ricque-Marie \& L. E. CruzSuárez (Eds.) Avances en nutrición acuícola IV. Memorias del IV Simposium Internacional de Nutrición Acuícola. Noviembre 15-18, La Paz, México.

Osuigwe, D. I., A. I. Obiekezie, J. O. Ogunji. 2002. Preliminary evaluation of Jack bean (Canavalia ensiformis) seed meal as a substitute for fishmeal in diets for Clarias gariepinus In: Challenges to organic farming Deutscher Tropentag. Oct. 9-11, 2002. Kassel Witzenhause.

Pérez, J. J., G. A. Wicki, F. J. Moyano, F. J. Alarcón. 2003. Evaluación del efecto de inhibidores de proteasa presentes en ingredientes vegetales utilizables en piensos para dos especies piscícolas cultivadas en Argentina; Pacú (Piaractus mesopotamicus) y Pejerrey (Odontesthes bonaeriensis). II Congreso Iberoamericano Virtual de Acuicultura. CIVA 2003: 442-454.
Rodiles, A., E. Santigosa, M. Herrera, I. Hachero-Cruzado, M. L. Cordero, S. Martínez-Llorens. 2012. Effect of dietary protein level and source on digestive proteolytic enzyme activity in juvenile Senegalese sole, Soleas enegalensis Kaup 1850. Aquaculture International 20: 10531070.

Rosenthal, G. A. 1992. Non-Protein amino acids in the life processes of higherplants. pp. 249-261. In: Singh, B. K. (ed.), Biosynthesis and Molecular Regulation of AminoAcids in Plants. American Society of Plant Physiologists. Rockville, USA.

Saunders, R. M., M. A. Conner, A. N. Booth, E. M. Bickoff, G. O. Kohler.1972. Measurement of digestibility of alfalfa concentrates by in vivo and in vitro methods. Journal of Nutrition 103: 530-535.

Tacon, A. G. J., J. L. Webster, C. A. Martinez. 1984. Use of solvent extracted sunflower seed meal in complete diets for fingerling rainbow trout (Salmo gairdneri Richardson). Aquaculture 43: 381-389.

Tiamiyu, L. O., V. T. Okomoda, P. O. Akpa. 2016. Nutritional profile of toasted Canavalia ensiformis seed and its potential as partially replacement for soybean in the diet of Clarias gariepinus. Brazilian Journal of Aquatic Science and Technology 20(2): 12-17.

Thiessen, D. L., G. L. Campbell, P. D. Adelizi. 2003. Digestibility and growth performance of juvenile rainbow trout (Oncorhynchus mykis) fed with pea and canola products. Aquaculture Nutrition 9: 67-75.

Udedibie, A. B. I. 1990. Nutritional evaluation of jackbean (Canavalia ensiformis) for poultry industry in Nigeria. AMBIO 19: 361-365.

Udedibie, A. B. I., C. O. Nkwocha. 1990. Comparative study of jack bean (C. ensiformis) and sword bean (C. gladiata) as protein supplements for young broiler chicks. Agriculture Journal 24: 7-14.

Udensi, E. A., F. C. Ekwu, J. N. Isinguzo. 2007. Antinutrient factors of vegetable cowpea (Sesquipedalis) seeds during thermal processing. Pakistani Journal of Nutrition 6 (2): 194-197.

Urbano G., P., E. Aranda, A. Gómez-Villalva, J. M. Frejnagel Porres, J. Frías, C. Vidal-Valverde, M. López-Jurado. 2003. Nutritional evaluation of pea (Pisu mativum L.) protein diet after mild hydrothermal and with and without added phytase. Journal of Agricultural and Food Chemistry 51:2415-2420.

Walter, H. E. 1984. Proteinases: methods with hemoglobin, casein and azocoll as substrates. Pp. 270-277. Bergmeyern, H. J. (ed.) Methods of Enzymatic Analysis, vol. V. Verlag Chemie. Weinham, Germany.

Wyss, U., H. Bicjel. 1988. Ripe beans of Canavalia ensiformis (jack bean) as feed ingredient for monogastric animals. Animal Feed Science and Technology 20: 325-326. 